\title{
Response of the Ubiquitin-Proteasome System to Memory Retrieval After Extended-Access Cocaine or Saline Self-Administration
}

\author{
Craig T Werner', Mike Milovanovic', Daniel T Christian', Jessica A Loweth' and Marina E Wolf*, \\ 'Department of Neuroscience, Chicago Medical School at Rosalind Franklin University of Medicine and Science, North Chicago, IL, USA
}

\begin{abstract}
The ubiquitin-proteasome system (UPS) has been implicated in the retrieval-induced destabilization of cocaine- and fear-related memories in Pavlovian paradigms. However, nothing is known about its role in memory retrieval after self-administration of cocaine, an operant paradigm, or how the length of withdrawal from cocaine may influence retrieval mechanisms. Here, we examined UPS activity after an extended-access cocaine self-administration regimen that leads to withdrawal-dependent incubation of cue-induced cocaine craving. Controls self-administered saline. In initial experiments, memory retrieval was elicited via a cue-induced seeking/retrieval test on withdrawal day (WD) 50-60, when craving has incubated. We found that retrieval of cocaine- and saline-associated memories produced similar increases in polyubiquitinated proteins in the nucleus accumbens (NAc), compared with rats that did not undergo a seeking/retrieval test. Measures of proteasome catalytic activity confirmed similar activation of the UPS after retrieval of saline and cocaine memories. However, in a subsequent experiment in which testing was conducted on WDI, proteasome activity in the NAc was greater after retrieval of cocaine memory than saline memory. Analysis of other brain regions confirmed that effects of cocaine memory retrieval on proteasome activity, relative to saline memory retrieval, depend on withdrawal time. These results, combined with prior studies, suggest that the relationship between UPS activity and memory retrieval depends on training paradigm, brain region, and time elapsed between training and retrieval. The observation that mechanisms underlying cocaine memory retrieval change depending on the age of the memory has implications for development of memory destabilization therapies for cue-induced relapse in cocaine addicts.
\end{abstract}

Neuropsychopharmacology (20I5) 40, 3006-30I4; doi:I0.1038/npp.20I5.I56; published online I July 20I5

\section{INTRODUCTION}

Exposure to cues previously paired with cocaine leads to retrieval of cocaine memories, which can evoke cocaine craving. This contributes to long-term vulnerability to relapse in cocaine addicts. Studies in animal models have shown that drug memories become labile upon retrieval and must undergo reconsolidation to be maintained, suggesting that interfering with reconsolidation has therapeutic potential for treating addiction (Milton and Everitt, 2010; Torregrossa and Taylor, 2013). It is therefore important to understand mechanisms mediating memory retrieval and reconsolidation.

In addition to well-established roles for NMDA receptors and downstream signaling pathways (Torregrossa and Taylor, 2013), de novo protein synthesis is necessary for retrieval and/or reconsolidation of cocaine memories (Lee et al, 2006; Fuchs et al, 2009; Fan et al, 2010; Théberge et al, 2010; Bailey et al, 2012; Ren et al, 2013; Shi et al, 2014).

*Correspondence: Marina E Wolf, Department of Neuroscience, Chicago Medical School at Rosalind Franklin University of Medicine and Science, 3333 Green Bay Road, North Chicago, IL 60064, USA, Tel: +847578 8659, Fax: +847578 8515,

E-mail marina.wolf@rosalindfranklin.edu

Received 4 March 2015; revised 8 May 2015; accepted 26 May 20I5; accepted article preview online 5 June 2015
Recently, it was found that the ubiquitin-proteasome system (UPS) is also required for retrieval-induced destabilization of cocaine memories in a conditioned place-preference (CPP) paradigm, suggesting coordinated roles for protein synthesis and degradation (Ren et al, 2013). This is analogous to a more established role for the UPS in destabilization of fear memories after their retrieval in a fear conditioning paradigm (Lee et al, 2008; Jarome et al, 2011).

The CPP results of Ren et al (2013) suggest that interfering with UPS activity might prevent cocaine memory reconsolidation and thus reduce cue-induced craving and relapse. However, CPP measures drug reward, not craving. Craving is an affective state in humans; in laboratory animals, it can be inferred, in drug self-administration studies, from responding for drug or drug-associated cues (Lu et al, 2004). Nothing is known about UPS activation when memories are retrieved after cocaine selfadministration. Furthermore, Ren et al (2013) conducted tests for memory retrieval 1 day after training, whereas relapsetriggering memories in addicts are usually much older. It is well established that remote memories are more resistant to protein synthesis inhibitors than recent memories (eg, Milekic and Alberini, 2002; Frankland et al, 2006) but whether UPS involvement changes as memories age has never been tested.

To address these issues, we measured UPS activation upon retrieval of cocaine memories after an extended-access 
cocaine self-administration paradigm that leads to progressive intensification, or 'incubation', of cue-induced craving over the first months of withdrawal (Lu et al, 2004; Pickens et al, 2011). We compared UPS activation at early and late withdrawal times, when craving is low and high, respectively, in order to assess its relationship both to the age of the memory and the intensity of craving. We focused on the nucleus accumbens (NAc) because the expression of 'incubated' craving depends upon synaptic plasticity in this region (Conrad et al, 2008; Loweth et al, 2014). We also studied other regions-amygdala, prefrontal cortex (PFC) and dorsolateral striatum (DLS) -implicated in cue-induced cocaine seeking after withdrawal (Grimm et al, 2003; Fuchs et al, 2006; Lu et al, 2005a, b, 2007; See et al, 2007; Koya et al, 2009; Pacchioni et al, 2011; Lee et al, 2013; Ma et al, 2014; Pascoli et al, 2014).

\section{MATERIALS AND METHODS}

\section{Cocaine Self-Administration}

Male Sprague-Dawley rats (250-275 g on arrival; Harlan Laboratories, Indianapolis, IN) were maintained on a reverse light-cycle with food and water available ad libitum. After a week to acclimate, jugular catheters were implanted and rats were singly housed. After 5-7 days of recovery, selfadministration training was conducted on 10 consecutive days ( $6 \mathrm{~h}$ /day) in operant chambers equipped with two nosepoke holes. Responding in one hole (active) resulted in intravenous infusion of cocaine $(0.5 \mathrm{mg} / \mathrm{kg} / \mathrm{infusion}$ in a $100 \mu \mathrm{l} / \mathrm{kg}$ volume over $3 \mathrm{~s}$ ) or saline ( $32 \mu \mathrm{l} /$ infusion) on an FR1 schedule, paired with illumination of a cue light (for detailed descriptions, see Conrad et al, 2008; Loweth et al, 2014). Responding in the inactive hole had no consequences. After completion of training, rats were housed in home cages and handled several times per week. Some rats (no retrieval groups) were killed without further testing. Others were returned to the operant chamber at different withdrawal times for a 30-min test designed to elicit memory retrieval and measure cueinduced cocaine seeking. Tests were performed under extinction conditions, ie, responding in the active hole delivered the light cue but no infusion. Procedures were approved by our Institutional Animal Care and Use Committee.

\section{Tissue Processing}

After rats were decapitated, regions of interest were dissected from $2 \mathrm{~mm}$ coronal slices prepared with a brain matrix (ASI instruments, Warren, MI), as shown in Supplementary Figure 1, and frozen at $-80{ }^{\circ} \mathrm{C}$. For NAc, both core and shell subregions were collected because incubation is accompanied by similar synaptic plasticity in both subregions (McCutcheon et al, 2011). After subcellular fractionation (see Supplemental Materials and Methods), protein polyubiquitination was measured in the $\mathrm{P} 2$ fraction; in some experiments, the S2 fraction was collected to measure proteasome catalytic activity.

\section{Immunoblotting}

P2 fractions prepared from brain regions of individual rats (10-15 $\mu$ g protein/lane) were mixed 1:1 with $2 \times$ sample treatment buffer (161-0737, Bio-Rad) and analyzed by SDSPAGE and immunoblotting (Ferrario et al, 2010). Proteins tagged with a Lysine-48-linked polyubiquitin chain are recognized and bound by the S5a subunit of the proteasome for degradation (Newton et al, 2008). To quantify proteins tagged in this manner, blots were probed using an antibody that selectively recognizes the ubiquitin Lysine-48 linkage. We also measured protein levels of two UPS targets: Shank and N-ethylmaleimide-sensitive factor (NSF). Antibodies and detection methods are described in Supplementary Materials and Methods.

\section{Proteasome Assays}

Proteasome catalytic activity was measured using the 20S Proteasome Activity Assay Kit (APT280, Millipore; Supplementary Materials and Methods; Supplementary Figure 2).

\section{RESULTS}

\section{Behavioral Data and Tissue Preparation}

Rats were trained (6 h/day, 10 days) to nose-poke to receive intravenous infusions of saline or cocaine paired with a light cue (Figure 1a; main effect of treatment: $F_{(1,69)}=558.7$, $P<0.0001$ ). On withdrawal day (WD) 1 or after prolonged withdrawal (WD50-60; see next section for justification of this withdrawal period), we induced memory retrieval and assessed cue-induced cocaine-seeking by returning animals to the operant chambers for a 30-min test (Figure 1b). During the test, nose-pokes in the previously active hole (the operational measure of cue-induced craving in incubation of craving studies; Lu et al, 2004; Pickens et al, 2011) delivered the light cue but no cocaine infusion. Two-way ANOVA revealed a significant interaction of treatment and WD $\left.\mathrm{F}_{(2,51)}=4.98, P<0.05\right)$. Post-hoc comparisons revealed that cocaine rats responded more on WD51 or WD60 (Sidak's, $\left.{ }^{* * *} P<0.0001,{ }^{* *} P<0.001\right)$ than on WD1, demonstrating incubation of cue-induced craving. Nose-pokes in the inactive hole for the cocaine groups were low $(8.78 \pm 0.733$; mean \pm SEM). Interestingly, combining all saline/retrieval groups revealed that these animals responded significantly more in the previously active hole compared with the inactive hole (Figure $1 \mathrm{~b}$, inset; $t_{(56)}=2.09,{ }^{\star} P<0.05$ ), indicating that saline animals remember the light cue.

\section{Protein Polyubiquitination and UPS Target Proteins in the NAc after Prolonged Withdrawal}

Our first question was whether memory retrieval/cueinduced cocaine seeking following incubation is associated with UPS activation in the NAc. We selected a withdrawal period (WD50-60) when incubation has peaked (Lu et al, 2004) and neuroadaptations in the NAc that underlie expression of incubation have stabilized (Wolf and Tseng, 2012). After this withdrawal period, some rats were given a seeking/retrieval test and then killed 30 or $60 \mathrm{~min}$ after memory retrieval (retrieval groups; Figure $2 \mathrm{a}$ and $\mathrm{b}$ ). Timing of the retrieval experiments was based on results from other memory retrieval paradigms (Lee et al, 2008; Jarome et al, 2011; Ren et al, 2013). Other rats were taken from home 

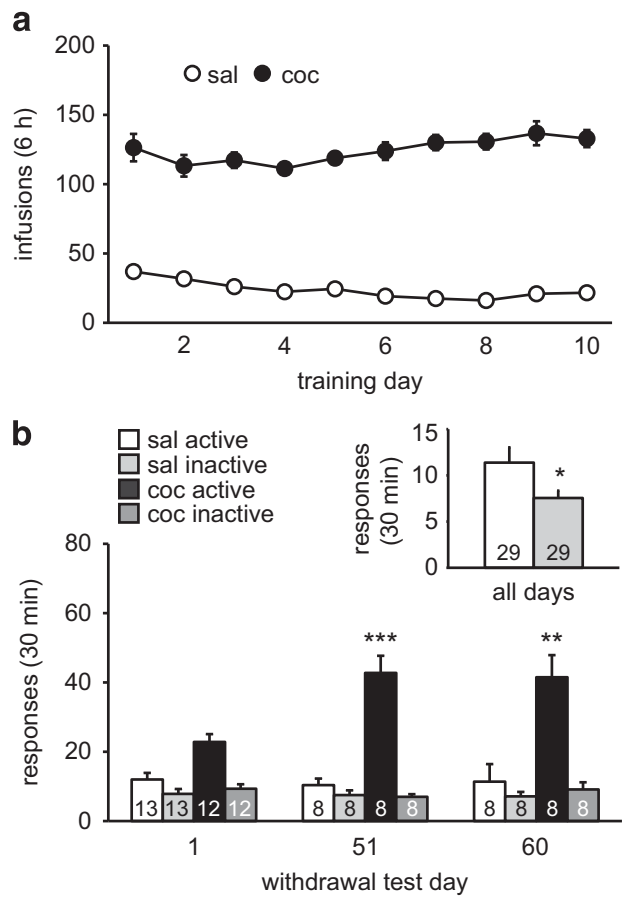

Figure I Self-administration training and cue-induced seeking/retrieval tests. (a) Training. Nose pokes in the active hole resulted in cocaine or saline infusions paired with a light cue. (b) Cue-induced seeking/retrieval tests. During the 30-min test, nose pokes in the previously active hole delivered the cue but no cocaine, providing a measure of cue-induced craving. Craving was increased ('incubated') in cocaine groups on both WD5I and WD60 compared with WDI. Inset: Combined results from all test days showed that saline rats responded significantly more in the previously active hole. $* P<0.05$, $* * P<0.0$ I, **** $P<0.00$ I. ANOVA followed by Sidak's multiple comparisons test. All data expressed as mean \pm SEM. The number of rats/ group in this and subsequent figures is shown within the bars.

cages for tissue collection without a seeking test (no retrieval groups) to determine if cocaine withdrawal is associated with altered basal UPS activity and to provide a baseline from which to assess seeking/retrieval test-induced changes in polyubiquitinated protein levels. After NAc tissue was harvested, the P2 synaptosomal membrane fraction, which is similar to fractions used in previous work (Lee et al, 2008; Jarome et al, 2011; Ren et al, 2013), was immunoblotted using an antibody recognizing polyubiquitinated proteins targeted for proteasomal degradation (see Materials and Methods). Samples from the retrieval and no retrieval groups were processed for SDS-PAGE and immunoblotting in parallel.

Comparing no retrieval groups to rats killed $30 \mathrm{~min}$ after starting the retrieval test (Figure 2c) by two-way ANOVA revealed a main effect of testing (retrieval $v s$ no retrieval: $\mathrm{F}$ $(1,28)=8.16, P<0.01)$ but no effect of prior treatment (saline vs cocaine; $\mathrm{F}(1,28)=0.25)$ and no significant interaction ( $\mathrm{F}$ $(1,28)=0.21)$. A similar pattern was observed when retrieval groups were killed $60 \mathrm{~min}$ after starting retrieval tests (Figure 2d; retrieval $v s$ no retrieval: $\mathrm{F}(1,28)=10.07$, $P<0.01$; saline $v s$ cocaine; $\mathrm{F}(1,28)=0.78$; interaction: $\mathrm{F}$ $(1,28)=0.17)$. Together, these results indicate that a seeking/retrieval test increases polyubiquitinated protein levels in the NAc regardless of whether a cocaine- or saline-associated memory is retrieved.
We considered the possibility that the retrieval-induced increase in polyubiquitinated proteins was due to exposure of both retrieval groups to a relatively novel context (it had been 50-60 days since their last exposure to the operant boxes). To address this, we used age-matched rats that were treatmentnaïve (they had remained in their home cages since entering the colony). These rats were either taken directly from their home cages for NAc tissue harvesting or first exposed to a novel context (the operant box) for $30 \mathrm{~min}$. We found no differences in levels of polyubiquitinated proteins between these groups (home cage: $100 \pm 3.69 \%$; novel context: $95.32 \pm 4.88 \%$; mean \pm SEM, \% control), suggesting that elevation of polyubiquitinated proteins in the cocaine/ retrieval and saline/retrieval groups was not due to renewed 'novelty' of the operant chamber after prolonged withdrawal.

We next measured total protein levels of two synaptic proteins, Shank and NSF. The UPS-mediated degradation of NSF, a protein whose functions include regulation of AMPA receptor trafficking (Hanley, 2014), has been linked to expression of cocaine CPP (Ren et al, 2013). The synaptic scaffolding protein Shank is tagged and degraded by the UPS after retrieval of fear memories (Lee et al, 2008; Jarome et al, 2011). At the 30-min time point, analysis of NSF levels by two-way ANOVA indicated that NSF was decreased in the retrieval groups compared with no retrieval groups (Figure 2e; main effect: retrieval $v s$ no retrieval: $\mathrm{F}(1,26)=5.55$, $P<0.05)$. Although there was no main effect of prior treatment (saline $v s$ cocaine: $\mathrm{F}(1,26)=0.60$ ), the saline retrieval group showed a trend toward a more pronounced decrease in NSF than the cocaine retrieval group (interaction: $\mathrm{F}(1,26)=1.71, P=0.20)$. The retrieval-induced decrease in NSF protein could reflect increased degradation by the UPS, based on increased polyubiquitinated protein levels at the same time point (Figure 2c), although other regulatory mechanisms could also account for altered NSF expression. Interestingly, the decrease in NSF observed $30 \mathrm{~min}$ after retrieval was transient, as indicated by no group differences in NSF levels at the 60-min time-point (Figure 2f; retrieval $v s$ no retrieval: $\mathrm{F}(1,28)=0.78$; saline $v s$ cocaine: $\mathrm{F}(1,28)=0.02$; interaction: $\mathrm{F}(1,28)=0.16)$.

Shank protein levels showed a retrieval-dependent but treatment-independent increase at both 30 -min (Figure $2 \mathrm{~g}$ ) and 60-min (Figure $2 \mathrm{~h}$ ) time points (30-min: retrieval $v s$ no retrieval: $\mathrm{F}(1,28)=15.74, \quad P<0.001$; saline vs cocaine: $\mathrm{F}$ $(1,28)=0.98$; interaction: $\mathrm{F}(1,28)=0.14 ; 60$-min: retrieval $v s$ no retrieval: $\mathrm{F}(1,28)=13.62, P=0.001$; saline $v$ s cocaine: $\mathrm{F}$ $(1,28)=0.47$; interaction: $F(1,28)=0.05)$. This could indicate decreased Shank degradation, although the same rats showed an increase in polyubiquitinated proteins suggestive of a general increase in protein degradation (Figure $2 \mathrm{c}$ and $\mathrm{d}$ ). An alternate possibility is that Shank translation is increased during memory retrieval, as changes in translation and degradation often go hand-in-hand during plasticity (Fonseca et al, 2006; Jarome et al, 2011).

\section{Polyubiquitinated Proteins and Proteasome Activity in Multiple Brain Regions after a Seeking/Retrieval Test during Prolonged Withdrawal}

We were surprised to observe similar UPS activation in saline/retrieval and cocaine/retrieval groups so we conducted an additional experiment to more thoroughly compare these 
a

\begin{tabular}{|c|c|c|}
$\begin{array}{c}\text { coc or sal } \\
\text { self-admin }\end{array}$ & withdrawal & seeking \\
\hline $6 \mathrm{~h} \times 10 \mathrm{~d}$ & $60 \mathrm{~d}$ & $\uparrow_{\text {retrieval }}^{30 \mathrm{~min}}$ \\
&
\end{tabular}

C
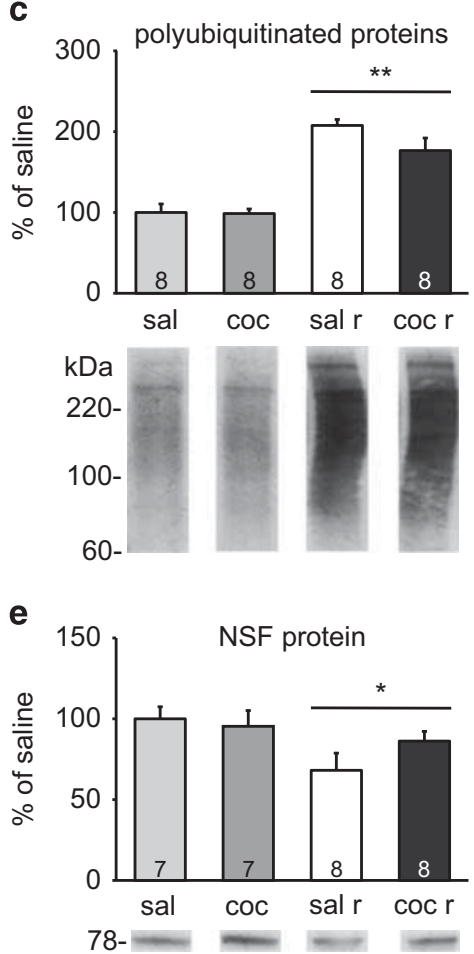

g

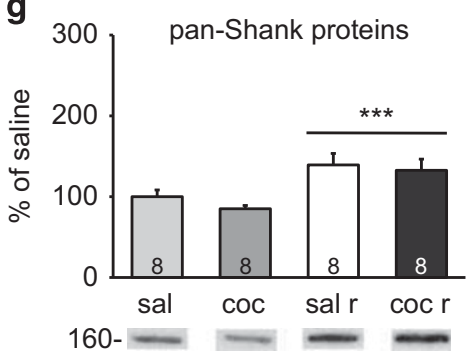

i

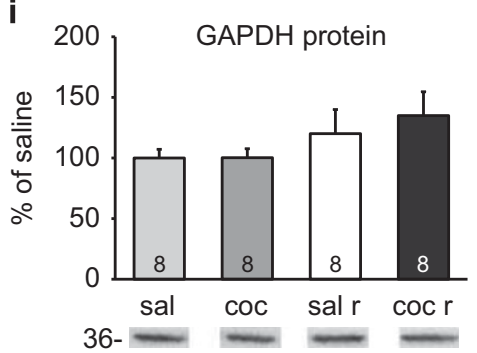

b

\begin{tabular}{ccc|c|} 
coc or sal & seeking home \\
self-admin & withdrawal & test & cage \\
\hline $6 \mathrm{~h} \times 10 \mathrm{~d}$ & $51 \mathrm{~d}$ & $\uparrow_{\text {retrieval }}^{30 \mathrm{~min}}$ & kill
\end{tabular}

d

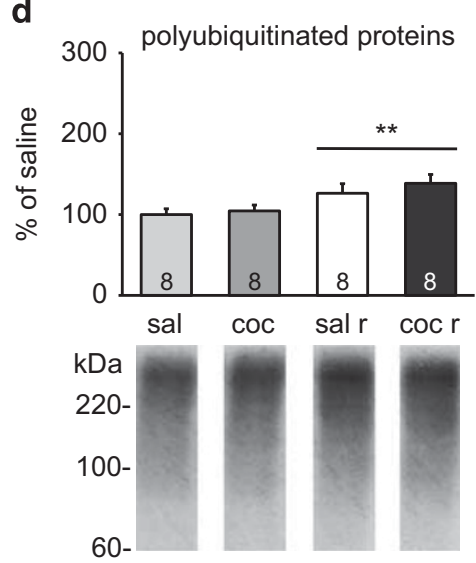

f

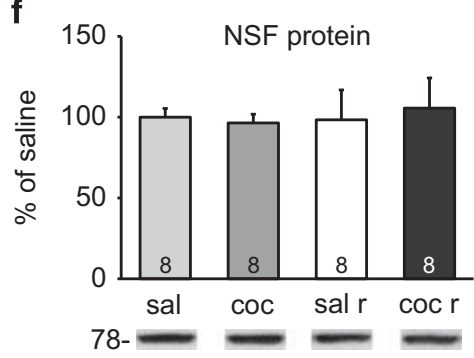

h

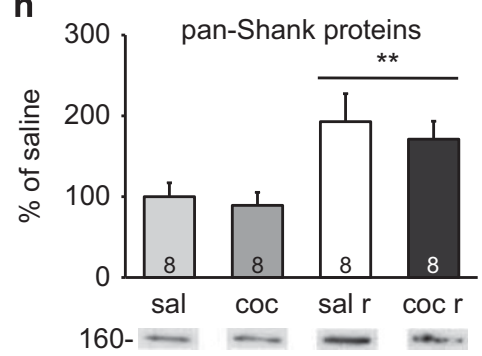

j

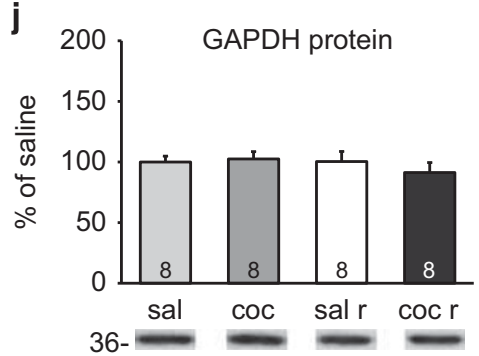

Figure 2 Polyubiquitinated proteins and protein targets of the proteasome in the NAc after prolonged withdrawal with or without a cue-induced seeking/ retrieval test. ( $a$ and b) Timelines: Saline/retrieval (sal r) and cocaine/retrieval (coc r) groups were killed either 30 min (a) or 60 min (b) after the start of a 30-min test. Saline/no retrieval (sal) and cocaine/no retrieval (coc) groups, not depicted on the timeline, were killed on WD50 without a seeking test. (c and d) In the NAc of rats killed 30 (c) or $60 \mathrm{~min}$ (d) after starting the test, there was a main effect of retrieval for polyubiquitinated proteins (** $P<0.0 \mathrm{I}$ ). NSF protein levels were decreased $30 \mathrm{~min}$ after retrieval in retrieval groups compared with no retrieval groups (e; *P<0.05) but returned to baseline 60 min after retrieval (f). pan-Shank immunoreactivity was increased $30(\mathrm{~g})$ and $60 \mathrm{~min}(\mathrm{~h})$ after retrieval (***P $<0.00 \mathrm{I}$, $* * P<0.0 \mathrm{I}$ ). (i and j) Levels of GADPH (loading control) did not differ significantly between groups. Data analyzed using two-way ANOVAs. 
a

\begin{tabular}{|c|c|}
\hline $\begin{array}{l}\text { coc or sal } \\
\text { self-admin }\end{array}$ & withdrawal seeking \\
\hline $6 \mathrm{~h} \times 10 \mathrm{~d}$ & $30 \mathrm{~min}$ \\
\hline
\end{tabular}

C
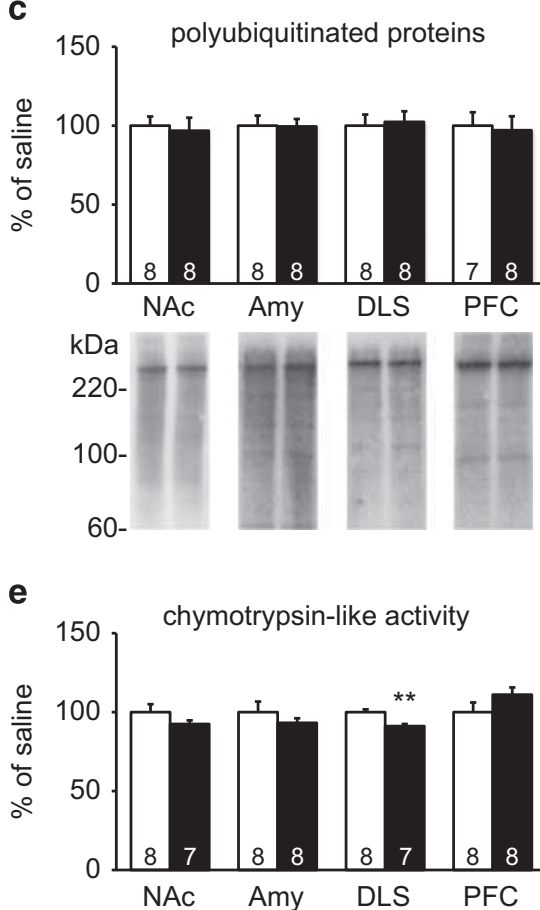

g

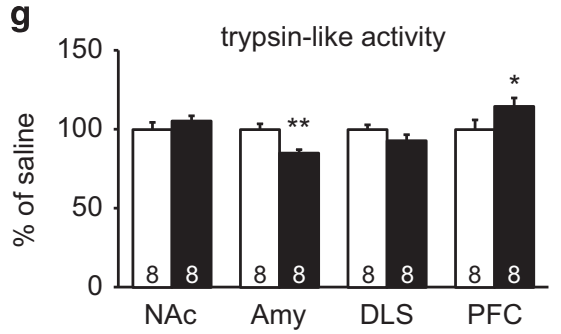

b

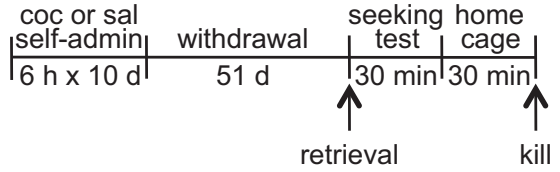

d

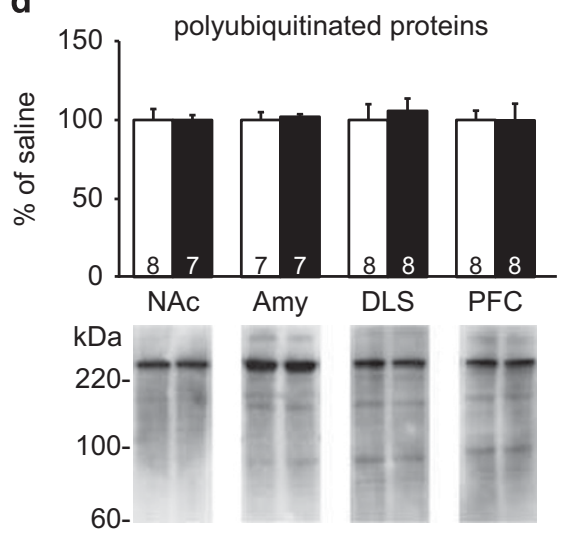

f

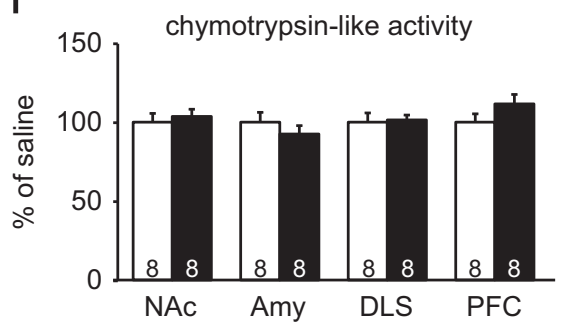

h

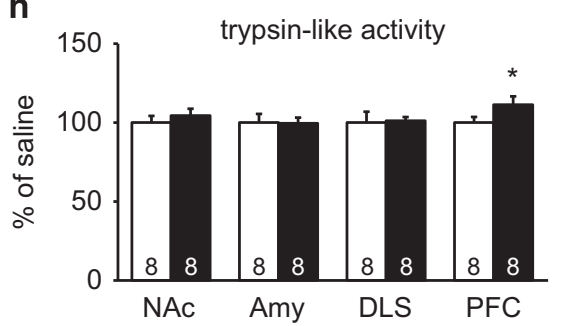

Figure 3 Polyubiquitinated proteins and proteasome activity following a cue-induced seeking/retrieval test during prolonged withdrawal. (a and b) All rats shown underwent a seeking/retrieval test after 5I-60 days of withdrawal and were killed either 30 min (a) or 60 min (b) after starting the test. (c and d) Polyubiquitinated protein levels did not differ between groups in any region at either time point. (e and f) Chymotrypsin-like activity of the proteasome was lower in the dorsolateral striatum (DLS) of cocaine/retrieval ( $\operatorname{coc} r$ ) rats compared with saline/retrieval (sal $r$ ) rats after 30 min $(* * P<0.0$ I) but no differences were found after $60 \mathrm{~min}$. ( $\mathrm{g}$ and $\mathrm{h}$ ) Trypsin-like activity was decreased in the amygdala (Amy) and increased in the prefrontal cortex (PFC) of cocaine/retrieval rats compared with saline/retrieval rats after 30 min. Trypsin-like activity remained increased in the PFC after 60 min. $* P<0.05$, **P $<0.01$. Immunoblots shown in Figures $3 \mathrm{c}, \mathrm{d}$ and $4 \mathrm{~b}$ appear different from those shown in Figures $2 \mathrm{c}$ and $\mathrm{d}$ because these experiments were conducted a year apart using different lots of anti-ubiquitin Lysine-48 antibody.

groups to better understand mechanisms underlying cocaine-specific memory retrieval, which has therapeutic relevance to cue-induced relapse. This experiment differed from our prior experiments in three ways: (1) it did not include 'no retrieval' groups, (2) in addition to preparing P2 fractions for analysis of protein polyubiquitination, we prepared S2 fractions for measurement of proteasome catalytic activity (Supplementary Materials and Methods; Supplementary Figure 2), and (3) we harvested DLS, amygdala, and PFC, in addition to NAc. The timing of the new experiment was identical to those shown in Figure 2.
Thus, after 50-60 days of withdrawal from saline or cocaine self-administration, rats received a cue-induced seeking/ retrieval test and were killed either $30 \mathrm{~min}$ (Figure 3a) or $60 \mathrm{~min}$ (Figure $3 \mathrm{~b}$ ) after starting the test. Based on previous work (Lee et al, 2008; Jarome et al, 2011), we hypothesized that the cocaine group would exhibit increased UPS activity in amygdala (and perhaps other regions) but found no differences in levels of polyubiquitinated proteins between saline/retrieval and cocaine/retrieval groups in any brain region at either time-point (Figure $3 \mathrm{c}$ and $\mathrm{d}$; all $t$-tests nonsignificant). For the NAc, this replicates the lack of difference 
between these groups observed in our earlier experiment (Figure $2 \mathrm{c}$ and $\mathrm{d}$ ).

Next, we measured proteasome catalytic activity in S2 fractions obtained from the same rats. We found no differences between cocaine/retrieval and saline/retrieval groups for proteasome activity in the NAc at either the 30min (Figure $3 \mathrm{e}$ and g) or 60-min (Figure $3 \mathrm{f}$ and $\mathrm{h}$ ) time point (all $t$-tests non-significant). However, compared with the saline/retrieval group, the cocaine/retrieval group (30-min time point) exhibited relatively less chymotrypsin-like activity in DLS but not in other regions (Figure 3e; DLS: $t_{(13)}=3.92, \quad{ }^{*} P<0.01 ;$ amygdala: $t_{(14)}=0.93$, NS; PFC: $t_{(14)}=1.45$, NS), as well as relatively less trypsin-like activity in the amygdala and more in the PFC (Figure 3g; amygdala: $t_{(13)}=3.50,{ }^{* *} P<0.01$; PFC: $t_{(14)}=1.80,{ }^{\star} P<0.05$, one-tail test; DLS: $t_{(14)}=1.45$, NS). In addition, compared with the saline/retrieval group, the cocaine/retrieval group showed greater trypsin-like activity in the $\mathrm{PFC}$ at the 60 -min time point (Figure 3h; PFC, $t_{(14)}=1.78,{ }^{*} P<0.05$, one-tail test; amygdala: $t_{(14)}=0.08$, NS; DLS: $t_{(14)}=0.15$, NS) but no significant differences in chymotrypsin-like activity in any region (Figure 3f; all $t$-tests non-significant). Thus, when a seeking/retrieval test is given after prolonged withdrawal, saline/retrieval and cocaine/retrieval groups do not differ with respect to NAc proteasome activity but show small, time-dependent differences when proteasome activity is measured in DLS, amygdala, and PFC. Therefore, proteasome activity in the latter regions depends to some degree on whether saline or cocaine memories are retrieved.

\section{Cocaine/Retrieval Rats Show Greater NAc Proteasome Activity than Saline/Retrieval Rats when Retrieval Occurs on WD1}

Our seeking/retrieval tests described above were conducted after many weeks of withdrawal, whereas all previous tests showing elevated UPS activity after memory retrieval were performed the day after completion of training (Lee et al, 2008; Jarome et al, 2011; Ren et al, 2013). To determine whether time elapsed between training and testing influences the UPS response, we examined polyubiquitinated protein levels and proteasome activity after a cue-induced seeking/ retrieval test on WD1 (the day after training finished). Tissue was collected $60 \mathrm{~min}$ after starting the test (Figure 4a), a time point when polyubiquitinated proteins were increased in the NAc (Ren et al, 2013), amygdala (Jarome et al, 2011), and hippocampus (Lee et al, 2008) following memory retrieval in other paradigms. All other aspects of this experiment were identical to experiments shown in Figure 3.

Analysis of polyubiquitinated protein levels revealed a significant increase in the cocaine/retrieval group compared with the saline/retrieval group for DLS but not other regions, although a trend was found for NAc (Figure 4b; NAc: $t_{(23)}=1.29, P=0.21$; DLS: $t_{(23)}=2.25,{ }^{\star} P<0.05$; amygdala: $t_{(23)}=1.46$, NS; PFC: $t_{(22)}=0.18$, NS). However, in proteasome activity assays, we found significant increases in chymotrypsin-like activity (Figure $4 \mathrm{c} ; t_{(24)}=1.96,{ }^{*} P<0.05$, one-tail test) and trypsin-like activity (Figure $4 \mathrm{~d} ; t_{(23)}=2.06$, ${ }^{\star} P<0.05$, one-tail test) in the NAc in the cocaine/retrieval group compared with the saline/retrieval group. These effects were not observed when the seeking/retrieval test was performed after prolonged withdrawal (Figure $3 \mathrm{e}-\mathrm{h}$ ). In a
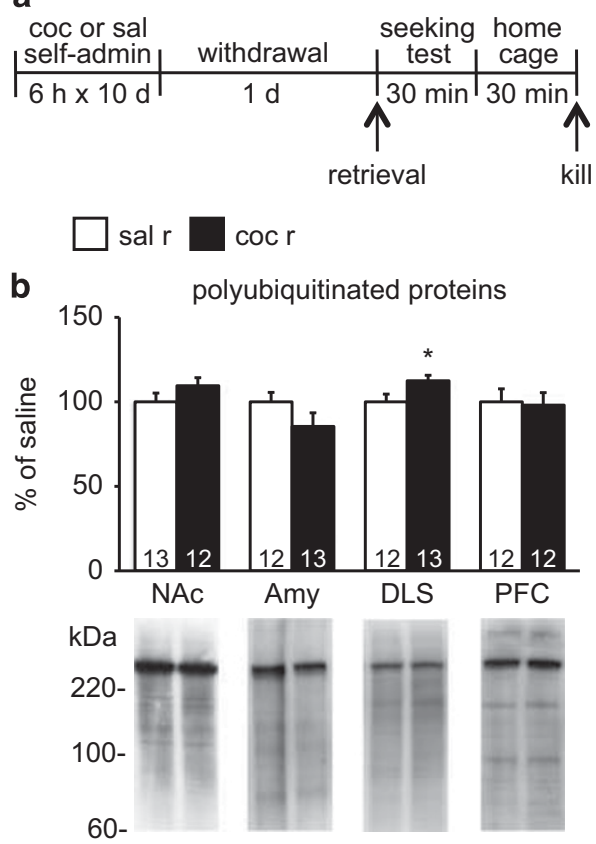

c
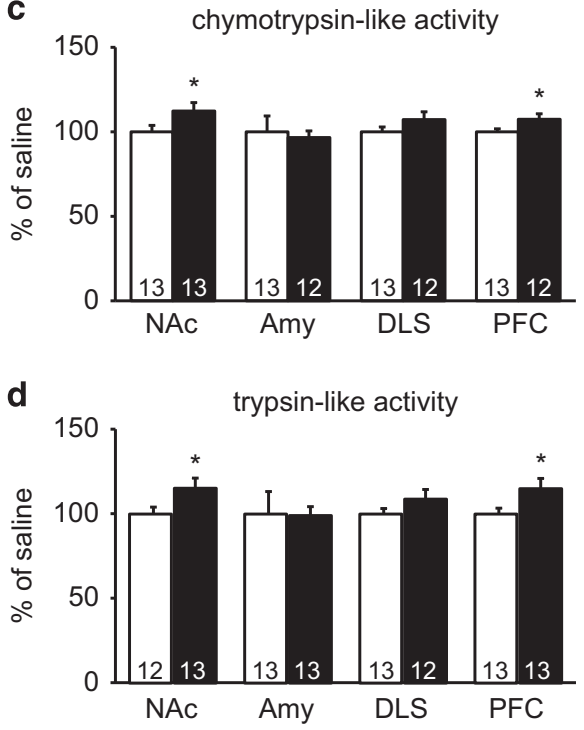

Figure 4 Polyubiquitinated proteins and proteasome activity following a cue-induced seeking/retrieval test on WDI. (a) Rats were killed 60 min after a seeking/retrieval test. (b) Polyubiquitinated protein levels were increased in the DLS of cocaine/retrieval rats compared with saline/retrieval rats. (c) Chymotrypsin-like and (d) trypsin-like activities of the proteasome were greater in the NAc and PFC of cocaine/retrieval rats. $* P<0.05$.

addition, the cocaine/retrieval group showed significant increases in chymotrypsin- and trypsin-like activity in PFC (chymotrypsin: $t_{(23)}=2.05,{ }^{\star} P<0.05$, one-tail test; trypsin: $t_{(24)}=2.15,{ }^{*} P<0.05$ ) but not amygdala or DLS (Figure 4c and $\mathrm{d}$; all $t$-tests non-significant). These results indicate that the interval between training and testing is an important determinant of UPS activation associated with memory retrieval. They also reveal a mismatch between changes in polyubiquitinated protein levels and proteasome activity. In general, one would expect increased polyubiquitinated proteins to precede and/or overlap with increased 
proteasome activity (eg, Jarome et al, 2013). A possible explanation for observing increased proteasome activity in the absence of increased polyubiquitinated protein levels (eg, compare NAc results in Figure $4 \mathrm{~b}-\mathrm{d}$ ) is that polyubiquitinated proteins increased during the seeking test, leading to an increase in proteasome activity after the test (when tissue was harvested). Due to this activity of the proteasome, polyubiquitinated proteins may have been degraded and returned to control levels by the time tissue was harvested. Although it would be interesting to test this possibility by performing an earlier seeking test, proteasome catalytic activity is the more direct measure of UPS activation so our results are sufficient for the between-group comparisons made here.

\section{DISCUSSION}

Protein degradation via the UPS is important in synaptic plasticity (Ehlers 2003; Bingol and Sheng, 2011; Li and Man, 2013), including plasticity following retrieval of fear (Lee et al, 2008; Jarome et al, 2011) and drug (Massaly et al, 2013; Ren et al, 2013) memories. However, no studies have examined its role in drug memory retrieval following drug selfadministration or at different times after the last drug exposure. The goal of the present study was to fill this gap using an extended-access cocaine self-administration regimen, in which cocaine-exposed rats show incubation of cueinduced craving over the first 1-2 months of withdrawal (Lu et al, 2004). First, we measured basal UPS activity after a period of withdrawal (WD50-60) when craving has incubated and stabilized at high levels (Lu et al, 2004). The NAc was studied because neuroadaptations in this region are required for expression of incubation after prolonged withdrawal (Conrad et al, 2008; Loweth et al, 2014). Importantly, these neuroadaptations are very stable from WD30-70, further justifying our selection of the WD50-60 period (Wolf and Tseng, 2012), and can be detected under basal conditions (ie, they do not require a seeking test to be evident). Therefore, we hypothesized that basal UPS activity might be altered in the NAc of 'incubated rats'. Contrary to this hypothesis, we found no basal difference in protein polyubiquitination between cocaine and saline rats that did not receive a seeking test (no retrieval groups). Therefore, the first conclusion from our study is that, despite evidence that drugs of abuse can influence the UPS (Massaly et al, 2013), incubation of craving does not alter basal UPS function in the NAc.

Next, we tested the hypothesis that memory retrieval would produce stronger effects on UPS activity in the NAc of 'incubated rats' (WD50-60) compared with saline controls. To do this, we compared protein polyubiquitination under basal conditions (no retrieval groups) to identically treated rats that received a seeking/retrieval test (saline/retrieval and cocaine/retrieval groups). To our surprise, the test similarly increased polyubiquitinated proteins in the NAc independent of whether saline or cocaine memories were retrieved, despite the fact that a cocaine-paired cue clearly drives a more powerful behavioral response than a saline-paired cue. Likewise, two UPS target proteins, Shank and NSF, were similarly affected by saline and cocaine memory retrieval, although there was a trend toward a less pronounced effect on NSF in the cocaine/retrieval group. Thus, the second conclusion of our study is that it may be difficult to selectively affect cocaine memories by targeting the UPS, although further studies are necessary to explore this. Recruitment of similar synaptic mechanisms upon retrieval of cocaine and saline memories makes sense if retrieval is only the first step in enabling a memory to engage the NAc mechanisms that convert 'motivation to action' (Mogenson et $a l, 1980)$ and suggests that cocaine-specific adaptations affecting steps downstream of retrieval (eg, strengthening of AMPA receptor transmission; Conrad et al, 2008; Loweth et al, 2014) explain the stronger behavioral response to cocaine-paired cues after prolonged withdrawal.

In our final series of studies, we conducted a more detailed comparison of saline/retrieval and cocaine/retrieval groups. We measured protein polyubiquitination and proteasome activity, evaluated WD1 and prolonged withdrawal, and analyzed amygdala, DLS, and PFC, as well as NAc. The most striking result was that, compared with the saline/retrieval group, the cocaine/retrieval group showed a seeking testinduced increase in NAc proteasome activity on WD1 that was not observed on WD50-60. Other brain regions also displayed different patterns of UPS activation on WD1 vs WD50-60. Thus, the third major conclusion is that the time elapsed between training and memory retrieval is an important determinant of retrieval-associated UPS activation. Whether this last conclusion applies to other paradigms remains to be determined. All prior studies of UPS activation accompanying memory retrieval used Pavlovian tasks (fear conditioning, or CPP after a limited number of noncontingent cocaine exposures), were conducted $24 \mathrm{~h}$ after completion of training, and did not include control groups in which UPS activity was measured after a non-drug or nonfear memory was retrieved (Lee et al, 2008; Jarome et al, 2011; Ren et al, 2013). In contrast, cocaine self-administration is an operant task, drug exposure is contingent and extensive $(6 \mathrm{~h} /$ day), testing is often during prolonged withdrawal when many synaptic changes have already manifested, and saline memory can be assessed (Figure 1b).

When interpreting results of our WD1 experiment, it must be kept in mind that this experiment did not include 'no retrieval' groups. Therefore, although we can compare proteasome activity in cocaine/retrieval vs saline/retrieval rats, we do not know how much these groups may have increased over their 'no retrieval' baseline. The exception is the NAc, where we found no difference in basal protein polyubiquitination between saline/no retrieval and cocaine/ no retrieval groups, indicating that saline/retrieval and cocaine/retrieval groups start from the same baseline. Keeping this caveat in mind, our results do not support a correlation, for any of the brain regions studied, between the level of UPS activation and the intensity of cocaine craving during a seeking/retrieval test. As discussed above, cocaine/ retrieval rats show greater NAc proteasome activity than saline/retrieval rats on WD1 but no difference on WD50-60. This contrasts with a more critical role for NAc excitatory transmission in the expression of incubation after prolonged withdrawal (Conrad et al, 2008; Loweth et al, 2014). The DLS is important for cocaine seeking after withdrawal (Fuchs et al, 2006; See et al, 2007) but its inactivation decreases context-induced cocaine seeking after extended-access cocaine self-administration in a manner that is not clearly 
related to withdrawal time (Pacchioni et al, 2011). Therefore, the fact that cocaine/retrieval and saline/retrieval groups showed a small difference in DLS UPS activity during prolonged withdrawal (Figure 3e), but not on WD1 (Figure $4 \mathrm{c}$ and d), is likely unrelated to incubation. In the PFC, cocaine/retrieval rats exhibited slightly greater proteasome activity regardless of test day, perhaps reflecting the PFC's major role in regulating cocaine seeking across experimental models (Kalivas and Volkow, 2005). However, projections originating from different PFC subregions undergo distinct adaptations during withdrawal that can both promote and oppose incubation of craving (Koya et al, 2009; Ben-Shahar et al, 2013; Ma et al, 2014). Therefore, future studies should measure UPS activity in PFC subregions. In the amygdala, results on WD1 and most results after prolonged withdrawal suggested similar activation of the UPS regardless of whether saline or cocaine memories were retrieved. However, as in prior studies of UPS activation after fear conditioning (Jarome et al, 2011), our dissection did not differentiate subregions of the amygdala. Although the basolateral amygdala has a key role in cocaine memory reconsolidation (eg, Lee et al, 2006), it is the central nucleus that is strongly implicated in the incubation of cocaine craving ( $\mathrm{Lu}$ et al, 2005a,b, 2007; also see Lee et al, 2013).

More work is required to understand the functional role of the UPS in retrieval of memories that elicit cocaine craving. It will also be important to uncover molecular mechanisms linking cocaine to UPS regulation (eg, Shen et al, 2007). However, our study - the first to examine the role of the UPS in retrieval of cocaine memories following cocaine selfadministration, as well as the first to examine the UPS following retrieval in any operant learning paradigm or after a delay between training and testing-provides an important framework for future studies by revealing that the involvement of the UPS in memory retrieval depends greatly upon paradigm, brain region, and the age of the memory.

\section{FUNDING AND DISCLOSURE}

This research was supported by DA015835, DA009621, and K05 DA029099 (MEW); predoctoral NRSA DA036950 (CTW); postdoctoral NRSA DA036963 (DTC); and K99 DA038110 (JAL). The authors declare no conflict of interest.

\section{REFERENCES}

Bailey J, Ma D, Szumlinski KK (2012). Rapamycin attenuates the expression of cocaine-induced place preference and behavioral sensitization. Addict Biol 17: 248-258.

Ben-Shahar O, Sacramento AD, Miller BW, Webb SM, Wroten MG, Silva HE et al (2013). Deficits in ventromedial prefrontal cortex group 1 metabotropic glutamate receptor function mediate resistance to extinction during protracted withdrawal from an extensive history of cocaine self-administration. J Neurosci 33: 495-506.

Bingol B, Sheng M (2011). Deconstruction for reconstruction: the role of proteolysis in neural plasticity and disease. Neuron 69: $22-32$.

Conrad KL, Tseng KY, Uejima JL, Reimers JM, Heng LJ, Shaham Y et al (2008). Formation of accumbens GluR2-lacking AMPA receptors mediates incubation of cocaine craving. Nature 454: $118-121$.
Ehlers MD (2003). Activity level controls postsynaptic composition and signaling via the ubiquitin-proteasome system. Nat Neurosci 6: 231-242.

Fan HY, Cherng CG, Yang FY, Cheng LY, Tsai CJ, Lin LC et al (2010). Systemic treatment with protein synthesis inhibitors attenuates the expression of cocaine memory. Behav Brain Res 208: 522-527.

Ferrario CR, Li X, Wang X, Reimers JM, Uejima JL, Wolf ME (2010). The role of glutamate receptor redistribution in locomotor sensitization to cocaine. Neuropsychopharmacology 35: 818-833.

Fonseca R, Vabulas RM, Hartl FU, Bonhoeffer T, Nägerl UV (2006). A balance of protein synthesis and proteasome-dependent degradation determines the maintenance of LTP. Neuron 52: 239-245.

Frankland PW, Ding HK, Takahashi E, Suzuki A, Kida S, Silva AJ (2006). Stability of recent and remote contextual fear memory. Learning Mem 13: 451-457.

Fuchs RA, Bell GH, Ramirez DR, Eaddy JL, Su ZI (2009). Basolateral amygdala involvement in memory reconsolidation processes that facilitate drug context-induced cocaine seeking. Eur J Neurosci 30: 889-900.

Fuchs RA, Branham RK, See RE (2006). Different neural substrates mediate cocaine seeking after abstinence versus extinction training: a critical role for the dorsolateral caudate-putamen. J Neurosci 26: 3584-3588.

Grimm JW, Lu L, Hayashi T, Hope BT, Su TP, Shaham Y (2003). Time-dependent increases in brain-derived neurotrophic factor protein levels within the mesolimbic dopamine system after withdrawal from cocaine: implications for incubation of cocaine craving. J Neurosci 23: 742-747.

Hanley JG (2014). Subunit-specific trafficking mechanisms regulating the synaptic expression of $\mathrm{Ca}^{2+}$-permeable AMPA receptors. Semin Cell Dev Biol 27: 14-22.

Jarome TJ, Werner CT, Kwapis JL, Helmstetter FJ (2011). Activity dependent protein degradation is critical for the formation and stability of fear memory in the amygdala. PLoS ONE 6: e24349.

Jarome TJ, Kwapis JL, Ruenzel WL, Helmstetter FJ (2013). CaMKII, but not protein kinase A, regulates Rpt6 phosphorylation and proteasome activity during the formation of long-term memories. Front Behav Neurosci 7: 115.

Kalivas PW, Volkow ND (2005). The neural basis of addiction: a pathology of motivation and choice Am J Psychiatry 162: 1403-1413.

Koya E, Uejima JL, Wihbey KA, Bossert JM, Hope BT, Shaham Y (2009). Role of ventral medial prefrontal cortex in incubation of cocaine craving. Neuropharmacology 56: 177-185.

Lee JL, Milton AL, Everitt BJ (2006). Cue-induced cocaine seeking and relapse are reduced by disruption of drug memory reconsolidation. J Neurosci 26: 5881-5887.

Lee SH, Choi JH, Lee N, Lee HR, Kim JI, Yu NK et al (2008). Synaptic protein degradation underlies destabilization of retrieved fear memory. Science 319: 1253-1256.

Lee BR, Ma YY, Huang YH, Wang X, Otaka M, Ishikawa M et al (2013). Maturation of silent synapses in amygdala-accumbens projections contributes to incubation of cocaine craving. Nat Neurosci 16: 1644-1651.

Li AW, Man HY (2013). Ubiquitination of neurotransmitter receptors and postsynaptic scaffolding proteins. Neural Plast 2013: 432057.

Loweth JA, Scheyer AF, Milovanovic M, LaCrosse AL, Flores-Barrera E, Werner CT et al (2014). Synaptic depression via mGluR1 positive allosteric modulation suppresses cue-induced cocaine craving. Nat Neurosci 17: 73-80.

Lu L, Grimm JW, Hope BT, Shaham Y (2004). Incubation of cocaine craving after withdrawal: a review of preclinical data. Neuropharmacology 47: 214-226.

Lu L, Hope BT, Dempsey J, Liu SY, Bossert JM, Shaham Y (2005a). Central amygdala ERK signaling pathway is critical to incubation of cocaine craving. Nat Neurosci 8: 212-219.

Lu L, Dempsey J, Shaham Y, Hope BT (2005b). Differential longterm neuroadaptations of glutamate receptors in the basolateral 
and central amygdala after withdrawal from cocaine selfadministration in rats. J Neurochem 94: 161-168.

Lu L, Uejima JL, Gray SM, Bossert JM, Shaham Y (2007). Systemic and central amygdala injections of the mGluR(2/3) agonist LY379268 attenuate the expression of incubation of cocaine craving. Biol Pyschiatry 61: 591-598.

Ma YY, Lee BR, Wang X, Guo C, Liu L, Cui R et al (2014). Bidirectional modulation of incubation of cocaine craving by silent synapse-based remodeling of prefrontal cortex to accumbens projections. Neuron 83: 1453-1467.

Massaly N, Dahan L, Baudonnat M, Hovnanian C, Rekik K, Solinas $\mathrm{M}$ et al (2013). Involvement of protein degradation by the ubiquitin proteasome system in opiate addictive behaviors. Neuropsychopharmacology 38: 596-604.

McCutcheon JE, Wang X, Tseng KY, Wolf ME, Marinelli M (2011). Calcium-permeable AMPA receptors are present in nucleus accumbens synapses after prolonged withdrawal from cocaine self-administration by not experimenter-administered cocaine. J Neurosci 31: 5737-5743.

Milekic MH, Alberini CM (2002). Temporally graded requirement for protein synthesis following memory reactivation. Neuron 36 : 521-525.

Milton AL, Everitt BJ (2010). The psychological and neurochemical mechanisms of drug memory reconsolidation: implications for the treatment of addiction. Eur J Neurosci 31: 2308-2319.

Mogenson GJ, Jones DL, Yim CY (1980). From motivation to action: functional interface between the limbic system and the motor system. Prog Neurobiol 14: 69-97.

Newton K, Matsumoto ML, Wertz IE, Kirkpatrick DS, Lill JR, Tan J et al (2008). Ubiquitin chain editing revealed by polyubiquitinated linkage-specific antibodies. Cell 134: 668-678.

Pacchioni AM, Gabriele A, See RE (2011). Dorsal striatum mediation of cocaine-seeking after withdrawal from short or long daily access cocaine self-administration in rats. Behav Brain Res 218: 296-300.

Pascoli V, Terrier J, Espallergues J, Valjent E, O'Connor EC, Lüscher C (2014). Contrasting forms of cocaine-evoked plasticity control components of relapse. Nature 509: 459-464.

Pickens CL, Airavaara M, Theberge F, Fanous S, Hope BT, Shaham Y (2011). Neurobiology of the incubation of drug craving. Trends Neurosci 34: 411-420.

Ren ZY, Liu MM, Xue YX, Ding ZB, Xue LF, Zhai SD et al (2013). A critical role for protein degradation in the nucleus accumbens core in cocaine reward memory. Neuropsychopharmacology 38: 778-790.

See RE, Elliott JC, Feltenstein MW (2007). The role of dorsal vs ventral striatal pathways in cocaine-seeking behavior after prolonged abstinence in rats. Psychopharmacology 194: 321-331.

Shen H, Korutla L, Champtiaux N, Toda S, LaLumiere R, Vallone J et al (2007). NAC1 regulates the recruitment of the proteasome complex into dendritic spines. J Neurosci 27: 8903-8913.

Shi X, Miller JS, Harper LJ, Poole RL, Gould TJ, Unterwald EM (2014). Reactivation of cocaine reward memory engages the Akt/GSK3/mTOR signaling pathway and can be disrupted by GSK3 inhibition. Psychopharmacology 231: 3109-3118.

Théberge FR, Milton AL, Belin D, Lee JL, Everitt BJ (2010). The basolateral amygdala and nucleus accumbens core mediate dissociable aspects of drug memory reconsolidation. Learn Mem 17: 444-453.

Torregrossa MM, Taylor JR (2013). Learning to forget: manipulating extinction and reconsolidation processes to treat addiction. Psychopharmacology 226: 659-672.

Wolf ME, Tseng KY (2012). Calcium-permeable AMPA receptors in the VTA and nucleus accumbens after cocaine exposure: when, how, and why? Front Mol Neurosci 5: 72.

Supplementary Information accompanies the paper on the Neuropsychopharmacology website (http://www.nature.com/npp) 